\title{
An Overview of Controlling Vehicle Direction Using Brain Rhythms
}

\author{
Sweta VM ${ }^{1}$, Sunita P Sagat ${ }^{2}$, Manisha Mali ${ }^{3}$ \\ PG student, Department of Electronics and Telecommunication, N.B Navale Sinhgad College of Engineering, Solapur University,
} Maharashtra, India

Assistant Professor, Department of Electronics and Telecommunication, N.B Navale Sinhgad College of Engineering, Solapur University, Maharashtra, India

Assistant Professor, Department of Electronics and Telecommunication, N.B Navale Sinhgad College of Engineering, Solapur University, Maharashtra, India

\begin{abstract}
In recent years, lot many research activities have taken place in the field of EEG based BCI. Recent experiments have shown that this application finds good potential for disabled people where driving becomes an impossible task. This paper discusses potential of BCI application for controlling vehicle direction, its effectiveness and probable issues. This system works on EEG signals on MATLAB platform. This is implemented by signal acquisition, filtering, feature extraction and their classification on the basis of command given.
\end{abstract}

Keywords: BCI-Brain Computer Interface, EEG Electroencephalography, DWT-Discrete Wavelet Transform, SVM-support vector Machine, ZPF-Zero phase filtering.

\section{Introduction}

BCI is the natural way to augment human capabilities by providing a new interaction link with outside world and is particularly relevant as an aid for paralyzed humans, although it also opens up new possibilities in human robot interaction for able bodied people. One of these new fields of application is use of BCI in automatic way where driving becomes an impossible task for disabled people. In past couple of years BCI technology has expanded in leaps and bounds. This technology allows disabled person to move the wheel chair without touching wheels or pedals. Instead EEG sensor is programmed to pick up conscious thoughts like left or forward and wirelessly beamed them to custom built automated control system that pushes pedals and turns steering wheel. BCI monitors and acquires brain activity of user, analyses it to determine the intent of subject on the basis of frequency and cortical areas activated and finally transmits signals as executable commands.

Among different methods that are currently studied to monitor brain activity like Magneto encephalography (MEG), Functional magnetic resonance imaging (fMRI), Positron emission tomography (PET), Near infrared systems (NIRS) and Electro-encephalography (EEG), only EEG is currently promising mainly due to its portability, low power consumption and fine temporal resolution. Through this project a software platform is set up to support BCI applications whose performance could be implemented by signal acquisition, filtering, feature extraction and classification.

Several BCI systems have been developed based on electroencephalography (EEG), magneto encephalography (MEG), Functional magnetic resonance imaging (fMRI), and optical imaging [1]. However, MEG, fMRI, and optical imaging are technically demanding and expensive.

Furthermore, fMRI and optical imaging measure hemodynamic changes of blood flow and are less amenable to rapid communication [1]. Only EEG has the advantages of easy preparation, inexpensive equipment cost, and high temporal resolution in implementing BCIs. An EEG-based BCI system requires an elaborately designed task for generating reliable neural responses, a credible signal processing method for extracting particular signal features, and an efficient translation algorithm to produce output signals [2].

\section{Problem statement}

The main problem of disabled people is to move from one place to another place. The proposed system will control vehicle direction using EEG signals (brain rhythms) .The vehicle can be moved forward, backward, right and left using this system.

\section{Methodology}

The architecture of the system mainly consists of five components. Steps or the overview of proposed system is as shown in following figure. 


\section{International Journal of Science and Research (IJSR) \\ ISSN (Online): 2319-7064}

Index Copernicus Value (2013): 6.14 | Impact Factor (2014): 5.611

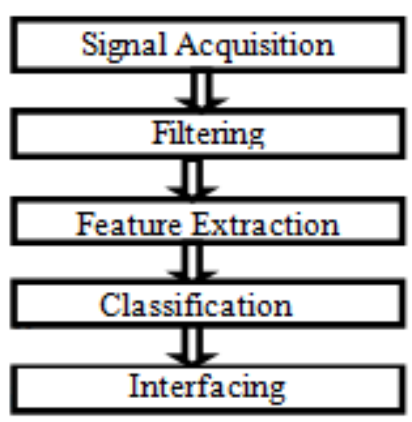

Figure 1 Overview of proposed system

\subsection{Signal Acquisition}

Electrical activity of the brain is measured by electrodes over the scalp. Electrodes establish connections between the scalp and EEG recording device by converting ionic current into electrical current. Electrolytic gel is applied between the scalp and the electrodes to prevent attenuation of the signal. An electrode placement system accepted as International standard called 10-20 System and is used to compare the measurements [4]. EEG measurement can be done in one of the two ways:

\section{a) Bipolar Recording}

C3-F3 and C4-F4 bipolar electrode pairs according to the 1020 System are used as two channels in this study in order to detect rhythms

\section{b) Referential Recording}

For SSVEP data acquisition, the positions of the electrodes are placed according to the International standard 10-20 system. The International 10-20 system is an internationally recognized method to describe and apply the location of scalp electrodes in the context of an EEG test or experiment. The first electrode, SSVEPPOS, is placed at the occipital region that is OZ (based on the International EEG 10-20 system); the second electrode, SSVEPNEG, is placed at the right mastoid; and the bipolar signal (SSVEPPOS to SSVEPNEG) with respect to the ground electrode (third electrode, SSVEPGND) is placed at the forehead position as shown in figure 2.

\section{SSVEP( GND)}

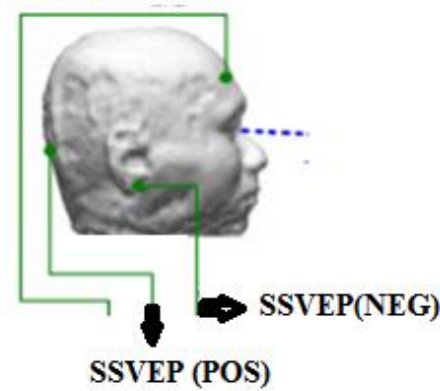

Figure 2 SSVEP Data Acquisition

\subsection{Filtering}

Acquired signals are usually contaminated with noises. The filtering method depends on the type of noises in signal. Various types of interferences in biomedical signals are from different origin. High frequency noise in EEG is due to instrumentation amplifier, recording system and EM signals by cables. Motion artifact in EEG may be caused in by coughing and breathing. Poor contact and polarization of electrodes may also cause low frequency artifacts. Due to harmonics distortion in fundamental frequency power line interference could appear in EEG which may be difficult to visualize.

First step in designing of filter is to remove the baseline noise. The baseline noise means the signal causes shifting due to movement. This shift has to be removed to get the original EEG signal. For removing the shift in EEG signal there are different techniques as follows.

i) FIR filtering

ii) IIR filtering

iii) Zero phase filtering

iv) Wavelet approach

v) Moving average approach

\subsubsection{FIR filtering}

FIR filters are digital fitters with finite impulse response. They are also known as non- recursive filter as they do not have feedback (a recursive part of a filter).even though the recursive algorithm can be used for FIR filter realization. The FIR filtering has an advantage of the stability as it has only zeroes rather than poles thus we do not need any specific location for them just like poles in IIR filter. FIR filter can be designed using different methods. Using window method is one of filtering method. The basic principle of the window design method is to truncate the ideal response with a finite length window .In the filter design we use different windows for truncating like Rectangular Window , Bartlett Window, Hanning Window, Hamming Window, and Blackman Window. The main lobe width is inversely proportional to the order of filter $\mathrm{N}$. An increase in the window length decreases the transition band of the filter. One of the drawbacks of FIR filter is high order of designed filter. The order of FIR filter is remarkably higher compared to an IIR filter with same frequency response. FIR filter is used only when linear phase characteristic is required

The designer must find a window with an appropriate side lobe level and then need to choose order to achieve the prescribed transition width for minimum stop band attenuation and pass band ripple. In this process the designer has to settle for a window with undesirable design specification and to overcome this problem Kaiser has chosen a class of windows which are based on the portable spheroidal function. The Kaiser window is given by following equation.

$$
W k=\frac{I 0\left[\alpha \sqrt{1-\left[\frac{2 n}{N-1}\right]^{2}}\right]}{I o(\alpha)} \text { for }|n| \leq \frac{N-1}{2}
$$

$=0$ otherwise

\subsubsection{IIR Filtering}

IIR filter are digital filters with infinite impulse response. 


\section{International Journal of Science and Research (IJSR) \\ ISSN (Online): 2319-7064}

Index Copernicus Value (2013): 6.14 | Impact Factor (2014): 5.611

Unlike FIR filter they have feedback (a recursive part of a filter) and are known as recursive digital filter thereof. The IIR filter transfer function is a ratio of two polynomials of complex variable $\mathrm{Z}-1$.The numerator defines location of zeroes and denominator defines location of poles of the resulting IIR filter transfer function

High pass filter are not at all pole filter and is given by

$$
H(s)=\frac{A h p * b s^{2}}{s^{2}+\frac{a}{b} W c S+\frac{W c^{2}}{b}}
$$

Where Ahp is high pass gain.

It should be noted that high pass filter are not at all pole filter as it contains twos s in numerator which shows that two zeroes are at origin. The frequency response of this filter decreases monotonically with frequency and can be given as

$$
|H(f=f c)|=\frac{1}{\sqrt{2}} \text { Where, } f_{c} \text { is cut-off frequency. }
$$

The decrease in frequency response is very slow in the pass band and quick in the stop band. In a design problem where no ripples are acceptable in case of pass band and stop band, Butterworth filter is a good choice, But it has nonlinear phase response and due to that waveform get distorted.

\subsubsection{Zero phase filtering(ZPF)}

The FIR filter has output combined with a group delay. As the filter order increases the complexity of the filter increases. The noise suppression performance of the filter will decrease if the selected filter order is low. IIR filter can achieve sharp transition region with a small number of coefficients. IIR filter that has a cut off frequency high enough to remove baseline noise. Because of nonlinear phase response of the IIR filter the distortion of meaningful components in the EEG waveform can happen. To avoid this distortion bidirectional filters are used in which the signal is filtered in a forward direction over a selected window and then same window is filtered in a reverse direction .A short window was preferably selected so that the filter could be used for real time purposes.

The delay of each frequency components is applied forwards and backwards in time and is therefore cancelled. Then the result has the following characteristics.

- Zero phase distortion.

- A filter transfer function which equals the squared magnitude of the original filter transfer function.

- A filter order that is double the order of the filter specified by numerator and denominator.

Zero phase filtering minimizes start up and ending transient by matching initial conditions. It helps in preserving the features in the filtered time waveform exactly where those features occur in the un filtered waveform .By using the coefficients of above discussed and implemented filters, FIR and IIR zero phase filtering is performed If the data in vector $\mathrm{X}^{\mathrm{G}}$ is filtered with the filter described by denominator vector $\underline{\underline{a}}^{6}$ and numerator vector $\underline{b}^{6}$ to create the filtered data $y^{6}$ the filter is described by the difference equation

$$
\begin{aligned}
& y[n]=a_{0} x[n]+a_{1} x[n+1]+a_{2} x[n+2]+a_{3} x[n+3]+\ldots \\
& b_{1} y[n+1]+b_{2} y[n+2]+b_{3} y[n+3]
\end{aligned}
$$

The above equation of recursive filter implemented in forward direction.

$$
\begin{aligned}
& y[n]=a_{0} x[n]+a_{1} x[n-1]+a_{2} x[n-2]+a_{3} x[n-3]+\ldots \\
& b_{1} y[n-1]+b_{2} y[n-2]+b_{3} y[n-3]
\end{aligned}
$$

The above equation is of reverse recursive filter implanted in backward direction. After the filtering in the forward direction the filtered sequence is the reversed and run back through the filter.

\subsubsection{Wavelet Approach}

A wavelet transform decomposes signal into basis function Which are known as wavelet transform and is calculated separately for different segments of the time domain signal At different frequencies resulting in a Multi resolution analysis. It is designed in such a way that the product of the time resolution and frequency resolution is constant. Therefore it gives good time resolution and poor frequency resolution at high frequency whereas good frequency resolution and poor time resolution at low frequency this feature of multi resolution analysis makes it excellent for signal having high frequency component for short duration and low frequency component for long duration.

Wavelet analysis consists of decomposing a signal into a hierarchical set of approximations and details. The words approximation and detail are justified by the fact that approximations taking into account the low frequencies whereas the details correspond to the high frequency correction [9]. As baseline wandering occurs at low frequencies so it is due to approximations. In this method the EEG signal is decomposed into 8 levels using Daubchies 6 wavelet. When the details are superimposed it result the waveform that eliminate the baseline drift . Different composition were also tried using approximation .but the inclusion of every approximation introduces the baseline drift.

\subsubsection{Moving Average Approach}

A moving average filter smooth's the data by replacing each data point with the average of the neighboring data points defined within the span. This process is equivalent to low pass filtering with the response of the smoothing given by the difference equation

$$
Y s(i)=\frac{1}{2 N+1}(Y(i+N)+Y(i+N-1)+\ldots+Y(i-N))
$$

where $\mathrm{Ys}(\mathrm{i})$ is the smoothed value for the ith data points , $\mathrm{n}$ is the number of neighboring data points on either side of Ys(i) and $2 \mathrm{~N}+1$ is the span[9].

By subtracting the output of this filter from original data, the data equivalent to high pass filtering can be achieved. The moving average smoothing method used by curve Fitting toolbox follows these rules [9] 


\section{International Journal of Science and Research (IJSR) \\ ISSN (Online): 2319-7064}

Index Copernicus Value (2013): 6.14 | Impact Factor (2014): 5.611

- The span must be odd

- The data points to be smoothed must be at the center of the span.

- The span is adjusted for data points that cannot accommodate the specified number of neighbors on either side.

The end points are not smoothed because a span cannot be defined.

\subsubsection{Notch Filter}

To remove the power line interference or noise present in EEG signal a notch filter $(50 \mathrm{~Hz})$ is used. A notch filter allows significant reduction of power line interference from measured signal. A filtered output signal has two components: a transient response and a steady state response. The measurement of the filtered output signal can be accelerated by reducing the transient response. The transient response duration strongly depends on the rejection bandwidth. Usually, if the rejection bandwidth is narrower, a longer transient response is observed. However, to reduce a sinusoidal interference without distortion in the frequency domain, notch filters with very narrow rejection bandwidth are chosen.

\subsection{Feature Extractions}

There are different feature extraction methods like principal component analysis, discrete derivatives, Wavelet Transform and FFT transform.

Wavelet transform is an effective way to extract features from non-stationary EEG signals. Through wavelet transform EEG signals are decomposed into frequency sub bands using discrete wavelet transform and a set for statistical features was extracted from the sub-bands to represent the distribution of wavelet coefficients according to the characteristics of signals.

$$
\begin{gathered}
W(a, b)=\int x(t) \psi *_{(a, b)}(t) d t \\
\psi_{a, b}(\mathrm{t})=\left(\frac{1}{\sqrt{a}}\right) \psi\left(\frac{(t-b)}{a}\right), a>0, b \in R
\end{gathered}
$$

where a and b are scale and translation parameters, respectively, $\Psi$ is the mother wavelet, ${ }^{*}$ is the complex conjugate operator and $\mathrm{W}(\mathrm{a}, \mathrm{b})$ is the continuous wavelet transform of $\mathrm{x}(\mathrm{t})$.

Scaled and translated versions of the basic functions are obtained from one prototype function, the mother wavelet. In principle, the CWT produces an infinite number of coefficients, thus it provides a redundant representation of the signal. The DWT provides a highly efficient wavelet representation that can be implemented with a simple recursive filter scheme and the original signal reconstruction can be obtained by an inverse filter [4]. The number of levels of decomposition is chosen on the basis of the dominant frequency components of the signal. According to the motor imagery EEG signals itself, level 4 and the wavelet of Daubechies order 10 was selected to extract $m$ and $b$ rhythms as features [4]. As a result, the EEG signal is decomposed into the details D1-D3 and approximation A3. The ranges of different frequency bands for a $128 \mathrm{~Hz}$ sampled signal are shown in table1.

Table 1: Frequencies correspond to different levels of decomposition for Daubechies order 10 wavelet with a sample rate $128 \mathrm{~Hz}$

\begin{tabular}{|c|c|c|}
\hline $\begin{array}{l}\text { Decomposed } \\
\text { Signal }\end{array}$ & $\begin{array}{c}\text { Frequency } \\
\text { range } \mathrm{Hz}\end{array}$ & Level \\
\hline D1 & $32-64$ & 1 \\
\hline D2 & $16-32$ & 2 \\
\hline D3 & $8-16$ & 3 \\
\hline A3 & $0-8$ & 3 \\
\hline
\end{tabular}

\subsection{Classification}

Extracted features of Wavelet coefficients for each trial can be classified depending upon the values obtained. A variety of classifiers have been used to translate these extracted features from EEG signals into an output command, from simple classifiers such as nearest neighbor, linear discriminant analysis (LDA), nonlinear neural networks (NN), support vector machines (SVM), and statistical classifiers.

LDA is a widely used linear classifier. Compared with the other methods, the main advantages of LDA include the following: 1) It is simple to use and 2) it has low computational complexity.

Artificial neural network (ANN) is a widely used nonlinear modeling method for regression analysis and classification, which is based on biological neural networks. The main advantage of ANN as a classification method is its ability to approximate arbitrary nonlinear decision functions by minimizing the error in classifying training data.

Unlike ANN, Support Vector Machine is one of the popular Machine Learning methods for classifying EEG signals. SVM aims to maximize the margin in order to avoid the risk of over fitting data and minimize the misclassification error SVM does not need to set up many configurations and parameters.

Another advantage of SVM is that it has good generalization characteristics and is especially suitable for the cases, where a small amount of training data is gained.

\section{Conclusion}

This paper introduces an overview of controlling vehicle direction using brain rhythms. The main topic discussed here is removing the artifacts from EEG signal by different approaches briefly, and an overview of different methods of classification and feature extraction.

\section{References}

[1] J. d. R. Mill'an, F. Renkens, J. Mourĩno, and W. Gerstner, Noninvasive brain-actuated control of a 
mobile robot by human EEG," IEEE Trans. Bio. Eng., vol. 51, no. 6, pp. 1026-1033, Jun. 2004)

[2] Luzheng $\mathrm{Bi}$, EEG based brain-controlled mobile robot:survey" IEEE Transactions On Human-Machine Systems, Vol. 43, No. 2, March 2013)

[3] Po-Lei Lee, Hsiang-Chih Chang, Tsung-Yu Hsieh, HuaTing Deng and Chia-Wei Sun" A Brain-Wave-Actuated Small Robot Car Using Ensemble Empirical Mode Decomposition-Based Approach" IEEE transactions on systems, man, and cybernetics-part a: systems and humans, vol. 42, no. 5, September 2012)

[4] Middendorf, M.S., McMillan, G., Calhoun, and G. und Jones, K.S. Brain-computer interfaces based on the steady-state visual-evoked response" Brain-Computer Interface Technology: Theory and Practice:1999, pp. 78-82)

[5] B G Xu and A G Song, Pattern Recognition of motor imagery EEG using wavelet transform," J. Biomedical Science And Engineering, vol. 1, pp. 64-67, 2008.)

[6] G. Pfurtscheller, C. Neuper, C. Guger, W. Harkam, H. Ramoser, A. Schlogl, B. Obermaier and M. Pregenzer, - Current trends in Graz Brain-Computer Interface (BCI) research," IEEE Trans. Rehab. Eng., vol. 8, no. 2, pp. 216-219, Jun. 2000)

[7] R. Palaniappan (\&) Department of Engineering, School of Science and Engineering, University of Wolverhampton, Telford, UK e-mail: palani@wlv.ac.uk;palani@ieee.org-Electroencephalogr am-based Brain-Computer Interface::An Introduction" (C) Springer-Verlag London 2014

[8] Sonali1, payal patial2 Đifferent Techniques of Baseline Wander Removal - A Review" Electronics and Communication Engineering, Lovely Professional University, India, International Journal of Enhanced Research in Science Technology \& Engineering, ISSN: 2319-7463 Vol. 2 Issue 5, May-2013, pp: (37-43)

[9] Matlab help, MATLAB MATHWORKS. http://www mathworks.com

\section{Author Profile}

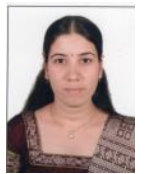

Sweta VM received the B.E (Electronics and tele communication) from Visvesvarayya Technological university ,Belagum. and M.E. degrees in Electronics and Tele communication Engineering from Sinhgad Institute of Technology in 2009 and 2016, respectively. During 2009-2012, she worked as hardware design engineer in iWave system Technologies PVT Ltd,Banglore

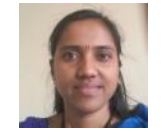

Prof S P Sagat received the B.E. (Electronics \& Telecommunication) \& M.E. Electronics- Digital Systems from University of Pune, Pune in 2001 and 2008 respectively. She is working as an Assistant Professor in Sinhgad College of Engineering, Solapur.

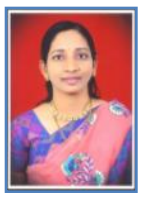

Prof M D Mali received the B.E(E\&TC) and M.E(E\&TC)in 2001 and 2011 respectively from Shivaji University, Kolhapur, Maharashtra. She is currently working as Assistant Professor in N B Navale Sinhgad College of engineering, Solapur. She has published 10 articles in professional journals and conferences. Her current research interests include computer vision, object recognition, image analysis, document processing, and computational geometry. 